\title{
A meta-analysis of Giardia lamblia in Iran
}

\author{
Laila Abasian ${ }^{1}$, Shahnaz shirbazou ${ }^{2}$, Fatemeh Talebi ${ }^{1}$, and Ali Delpisheh ${ }^{3,4 *}$ \\ ${ }^{1}$ Department of Parasitology, Ilam University of Medical Sciences, Ilam-Iran. \\ ${ }^{2}$ Health Research Center, Baqiyatallah University of Medical Sciences, Tehran, Iran. \\ ${ }^{3}$ Department of Epidemiology Ilam University of Medical Sciences, Ilam-Iran. \\ ${ }^{4}$ Clinical Microbiology Research Centre, Ilam University of Medical Sciences, Ilam-Iran.
}

Accepted 8 March, 2013

\begin{abstract}
Giardia lamblia is the frequent cause of diarrhea in humans and other mammals across the world. The present systematic review and meta-analysis aimed to estimate the epidemiology of Giardia lamblia in the republic Islamic of Iran. The main international sources of ISI and Pubmed as well as the major national data banks in Iran including Magiran, IranMedex, SID and IranDoc were systematically searched for the words" Giardia lamblia" since 1990 onward. Overall, 43 reliable and similar studies in terms of methodology were selected for a systematic review. A meta-analysis was also conducted using the random-effects model. Heterogeneity was investigated by the Cochran's $Q$ test. STATA was used for all analysis. Given the random- effects model, the prevalence of Giardia lamblia in Iran was estimated to be $14.7 \%$ (95\% reliability distance, $12.0 \%-16.6 \%$ ). By age classification, the prevalence was $15.1 \%$ amongst fewer than 10 years children, $19.2 \%$ amongst adolescents and youngest of fewer than 20 years, and $6.7 \%$ amongst adults of between $20-30$ years old $(p<0.001)$. Annual classification showed that the maximum and minimum prevalence rates occurred in 1994 and 2005 (37.21 and 4.91\% respectively). Geographical distribution of Giardia lamblia also showed that the eastern parts of the country nearby Pakistan and Afghanistan borders were extremely affected (35.5\%). It was $16.9 \%$ in central provinces, $14.9 \%$ in the southern provinces, $13.2 \%$ in the north of country and $12.3 \%$ in the western provinces nearby Iraq and Turkey borders. Increased prevalence of Giardia lamblia in Iran, in spite of preventing strategies and valuable primary health care activities has been remained as a public health concern amongst children and in the eastern parts of the country nearby Pakistan and Afghanistan borders in particular.
\end{abstract}

Key words: Giardia lamblia, Iran, meta-analysis.

\section{INTRODUCTION}

Traditionally, doctors and ordinary people have been focusing on parasitic infections and it has still remained among the important public health concerns leading to mass mortalities worldwide and in developing communities in particular. Infection with Giardia may be associated with significant ill-health issues and while the reported incidence of infection is of concern in Iran, the true prevalence of infection and extent of morbidity due to this organism is unknown.
Giardia Lamblia is an important human pathogen with a global distribution. This flagellate mono cellular is the pathogenic parasite of the upper part of the small intestine affecting many vertebrata including human (Disney et al., 1998; Baruch e al., 1996). The importance of potable water supplies as a source of infection is not obvious, nor is the role of zoonotic spread. Even though, water sources where polluted with the mature cysts of parasite are the main source of infection (Adam, 1991). 
Parasites are even survived for some times due to the relatively resistance of Giardia cysts to chlorine that is existed in the water (Sayyari and Imanzadeh, 2005).

The international reports shows a prevalence rate of 73.4\% in Nepal in 2004 (Easow et al., 2005), 11.6\% in Filipina (Baldo et al., 2004), 4.84\% in China (Yang et al., 2003), and 1.5\% in South Korea (Guk et al., 2005).

Although, Giardia lamblia affects all age groups but children are more vulnerable, sensitive and susceptible (Baruch et al., 1996), probably due to lack of immunesystem and personal health (Amar, 2000; Jahni et al., 2009). Prevention strategies include treatment of patients and disinfection of polluted drinking waters (Sayyari and Imanzadeh, 2005; Amar, 2000).

Iran is an appropriate environment for activation of various parasites due to the geographical features, the climatic conditions and the bio-cultural characteristics. Given that the recognition and controlling of risk factors of the parasitic infections, requires epidemiological investigations across the country. The present study aimed to investigate almost all studies conducted in Iran during the last decade regarding the Giardia lamblia infection.

\section{MATERIALS AND METHODS}

This was a systematic review and meta-analysis study on current investigations and research publications about epidemiology of Giardia lamblia in Iran during the last decade. The main international sources of ISI and Pubmed as well as the major national data banks in Iran including Magiran, Iran Medex, SID and Irandoc were systematically searched for the words" Giardia lamblia" since 1990 onward.

The summary of all 80 related papers was read carefully and those with similar methodologies $(n=43)$ were included(Mahyar 1997; Alavinaini 2000; Amar 2000; Abdolali 2001; Mahyar 2001; Mostafaei 2001; Rafiee 2001; Rohani 2001; Srasyabi 2001; Aminzadeh 2002; Farhnak 2002; Rohani 2002; Askari 2003; Davami 2003; Heidari 2003; Moghimi and Asghrshrifl 2003; Razavion 2003; Aminzadeh and Nazari 2004; Kheirandish 2004; Taheri 2004; Gholami 2005; Khalili 2005; Mohrez 2005; Sayyari and Imanzadeh 2005; Sharifi 2005; .J. 2006; Alborzi and Zerafati 2006; Atashnafas and Ghorbani 2006; Bahadori 2006; Hatmkhah 2006; Ashtiani 2008; Fallahi 2008; Ghofranipour 2008; Mahuti 2008; Mohsenimoghadam and Shahidizandi 2008; Molavi 2008; Monsef, Hashemi et al. 2008; Taherkhani 2008; Ghorbani 2009; Jahni, Rezaeian et al. 2009; Nasiri, Esmailnia et al. 2009 ; Mosallanejad, Avizeh et al. 2010; Taheri, Namakin K. et al. 2010) by a qualitative controlling method. The required information were extracted using a prepared check list. The original articles were provided and studied more in depth. Papers in which prevalence of Giardia lamblia in both genders were estimated regardless to the age and language of participants/patients were included. Unrelated papers and/or those with different methodologies /insufficient data were excluded from the study $(n=37)$. Of excluded articles, 20 did not meet the inclusion criteria and 17 articles were either repetitive or much localized. Decision upon either exclusion or inclusion of papers was independently made by two experienced reviewers.

All measurements for the meta-analysis were done using STATA software and random effects model. The heterogeneity was investigated by Cochran's $Q$ test. Figures were plotted using SPSS statistical software.

\section{RESULTS}

Meta-analysis model with random effects showed a prevalence rate of $14.7 \%$ (reliability distance of 95 - 16.6$12.8 \%$ ) for Giardia lamblia in Iran. The following age-specific prevalence rates were observed $(p<001)$ as demonstrated in Table 2:

1. $19.2 \%$ in the age group of $10-20$ years old (95\% reliability distance of $12.7-17.8$ )

2. $6.4 \%$ in the age group of $20-30$ years old (95\% reliability distance of $9.1-3.7$ )

3. $2.6 \%$ In the age group of $30-40$ years old (95\% reliability distance of 2.4-4.9)

The time trend of Giardia lamblia infection showed a maximum prevalence of $37.22 \%$ in 1994 and the least prevalence of $4.91 \%$ in 2005 (Figures 1 and 2; Table 1).

Geographical distribution of Giardia lamblia also showed that the eastern parts of the country nearby Pakistan and Afghanistan borders were extremely affected (35.5\%). It was $16.9 \%$ in central provinces, $14.9 \%$ in the southern provinces, $13.2 \%$ in the north of country and $12.3 \%$ in the western provinces nearby Iraq and Turkey borders (Table 3).

\section{DISCUSSION}

This is the first meta-analysis study in Iran as far as we have searched in the published literature, focusing on epidemiology of Giardia lamblia and its age-specific prevalence in particular. The descriptive cross sectional studies are the current ways for diseases assessments in the community. The present study found an overall prevalence rate of $14.7 \%$ for Giardia lamblia in Iran during the last decade starting from 1990 onward. Despite of this finding which put the Giardia lamblia as the most frequent intestinal parasite in Iran, but fortunately its annually trends in the whole country is decreasing.

In the current study, the maximum prevalence rate of $37.21 \%$ occurred in 1994 and amongst children and adolescents in particular by age-specific distribution analysis. The age-specific distribution analysis in the present study showed that Giardia lamblia was more frequent in the age lower than 20 years, while in the persons higher than 20 years, this age difference was not considerable. This could be in part related to unhealthy life style, consumption of low standard drinking water, resistance of Giardia cysts to chlorination and inappropriate health system due to rottenness of plumbs and flowing of sewages in some regions. Meanwhile, migrations, global warming, wars, natural disasters such as earthquake, floods, lack of health drinking water and resistance of the intestinal parasites to the treatment might be involved.

Even though, decreasing annual trends of Giardia lamblia in Iran could be related to the community health 


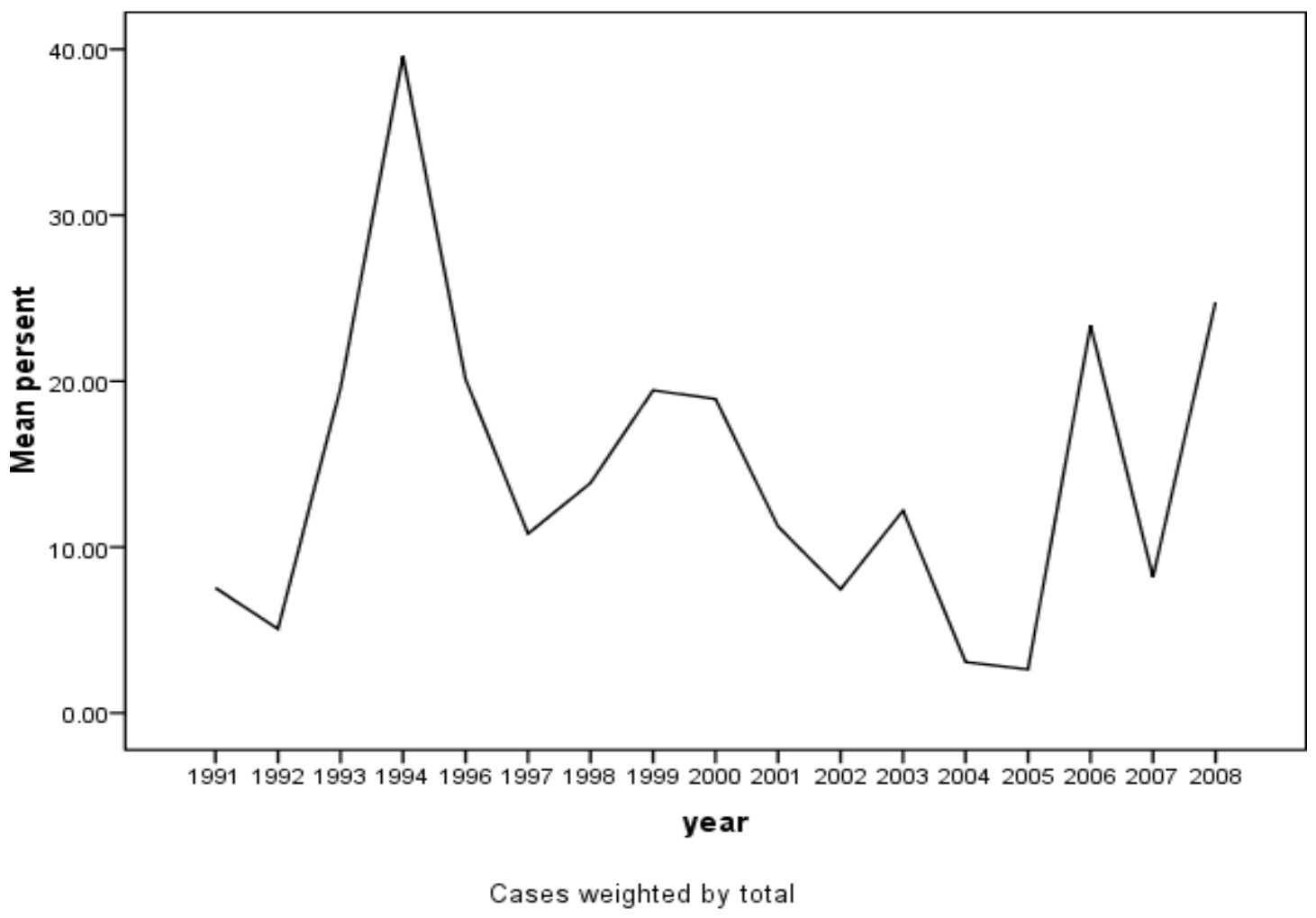

Figure 1. Annual trends of Giardia lamblia in Iran from 1990 onward.

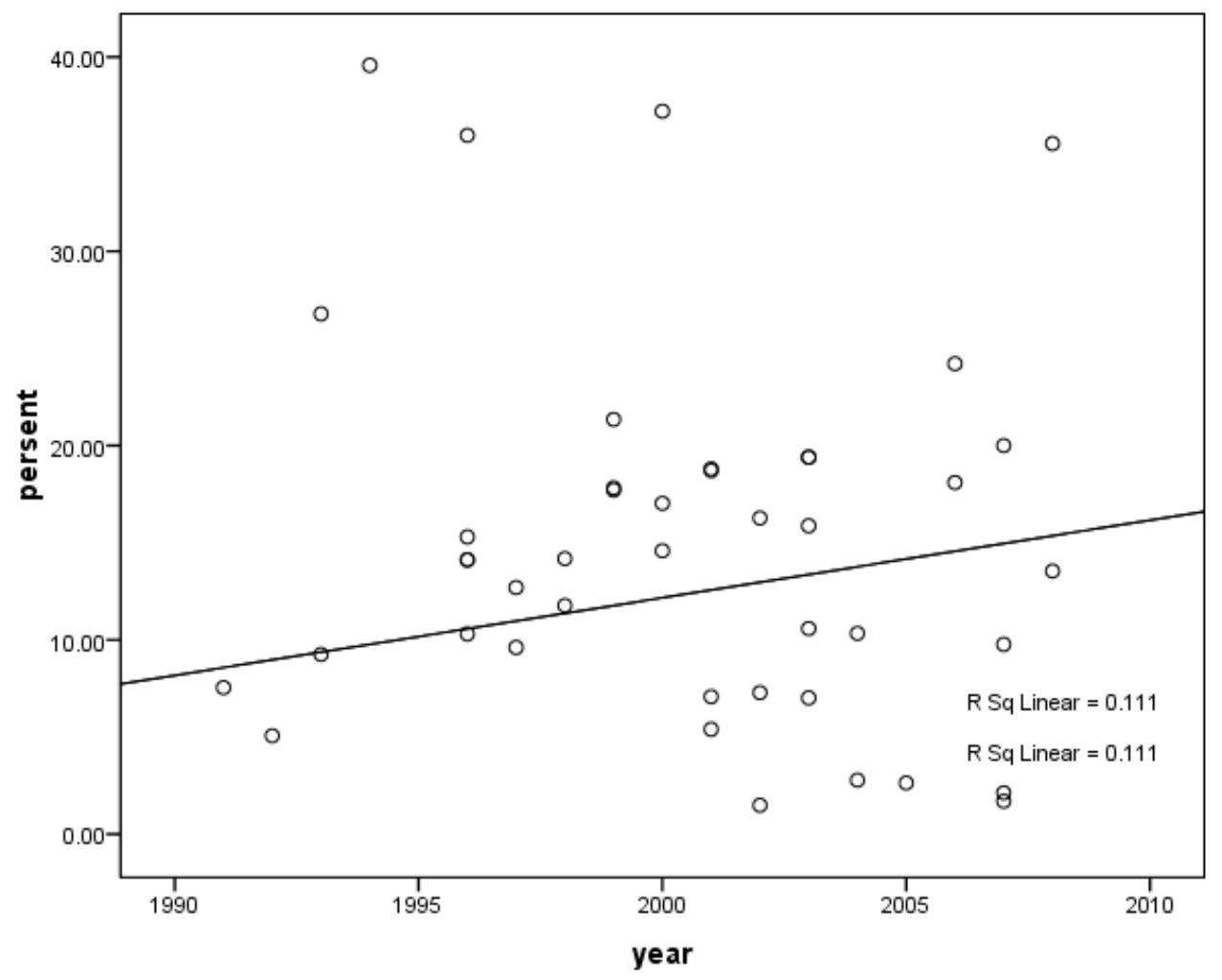

Figure 2. Regression plots of Giardia lamblia in Iran from 1990 onward. 
Table 1. Epidemiological characteristics of included studies on Giardia lamblia in Iran.

\begin{tabular}{|c|c|c|c|c|c|}
\hline Author & Year & Geographical situation* $^{*}$ & City & Sample size & Prevalence, \% \\
\hline Taheri & 2004 & $\mathrm{C}$ & Birjand & 10 & 1.48 \\
\hline Alborzi & 2007 & C & Shiraz & 17 & 1.70 \\
\hline Razavian & 2003 & $\mathrm{~N}$ & Mazandaran & 1 & 2.13 \\
\hline Jahani & 2009 & C & Tehran & 5 & 2.63 \\
\hline Bahadori & 2006 & W & Qaemshahre & 183 & 2.77 \\
\hline Srasyabi & 2001 & S & Hormozgan & 48 & 5.06 \\
\hline Davami & 2003 & W & Araak & 89 & 5.39 \\
\hline Heidari & 2003 & C & Dameghan & 75 & 7.01 \\
\hline Kheirandish & 2004 & W & Khoramabad & 66 & 7.07 \\
\hline Mohrez & 2005 & W & Kermanshah & 15 & 7.28 \\
\hline Ghorbani & 2009 & C & Tehran & 4842 & 7.54 \\
\hline Rohani & 2001 & $\mathrm{~N}$ & Noshahr & 38 & 9.25 \\
\hline Mahyar & 1997 & W & Qazvin & 39 & 9.61 \\
\hline Atashnafas and Ghorbani & 2006 & C & Semnan & 188 & 9.77 \\
\hline Mostafaei & 2001 & C & Kaashan & 37 & 10.31 \\
\hline Hatmkhah & 2004 & W & Urmia & 28 & 10.33 \\
\hline Gholami & 2005 & $\mathrm{~N}$ & Mazandaran & 171 & 10.86 \\
\hline Mahyar & 1997 & W & Ghazvin & 12 & 11.76 \\
\hline Farahnak & 2002 & W & Tabriz & 33 & 12.69 \\
\hline Gharavi & 2006 & W & Qazvin & 106 & 13.54 \\
\hline Hedayati et al. & 2008 & $\mathrm{C}$ & Tehran & 163 & 14.11 \\
\hline Mohsenimoghadam and Shahidizandi & 2008 & $\mathrm{E}$ & Rafsanjan & 163 & 14.11 \\
\hline Abdolali & 2001 & S & Yasooj & 95 & 14.18 \\
\hline Nasiri & 2009 & $\mathrm{C}$ & Karaj & 70 & 14.58 \\
\hline Alavinaini & 2000 & W & Saaveh & 145 & 15.30 \\
\hline Aminzadeh and Nazari & 2004 & C & Varamin & 40 & 15.87 \\
\hline Moghimi and Asghrshrifl & 2003 & $S$ & Yasooj & 75 & 16.27 \\
\hline Srasyabi & 2001 & S & Bandarabass & 236 & 17.03 \\
\hline Molavi & 2008 & C & Tehran & 440 & 17.13 \\
\hline Rohani & 2002 & $\mathrm{~N}$ & Saari & 222 & 17.82 \\
\hline Rafiee & 2001 & $\mathrm{C}$ & Tehran & 76 & 18.10 \\
\hline Khalili & 2005 & C & Yazd & 288 & 18.71 \\
\hline Sharif & 2001 & C & Yazd & 51 & 18.89 \\
\hline Fallahi & 2008 & W & Delfan & 97 & 19.40 \\
\hline Monsef & 2008 & W & Hamadan & 97 & 19.40 \\
\hline Mosallanejad & 2010 & $S$ & Ahvaz & 64 & 20.00 \\
\hline Amar & 2000 & $\mathrm{~N}$ & Babol & 735 & 21.43 \\
\hline Ashtiani & 2008 & C & Tehran & 642 & 24.99 \\
\hline Askari & 2003 & $S$ & Shiraz & 158 & 26.78 \\
\hline Taheri & 2010 & $E$ & Khorasan J. & 289 & 35.55 \\
\hline Mahuti & 2008 & W & Delfan & 500 & 35.97 \\
\hline Mahyar & 2001 & W & Qazvin & 96 & 37.21 \\
\hline Taherkhani & 2008 & W & Hamadan & 188 & 39.58 \\
\hline Total & & & & 10933 & 14.7 \\
\hline
\end{tabular}

N: Northern W: Western C: Central E: Eastern S: Sauterne* 
Table 2. Age-specific distribution of Giardia lamblia in Iran.

\begin{tabular}{ccccc}
\hline Age group (year) & Number of studies & Prevalence, $\%$ & $\mathbf{9 5} \% \mathbf{C l}, \%$ & Weight $^{*}$ \\
\hline $1-10$ & 22 & 15.1 & $12.4-17.8$ & 51.75 \\
$10-20$ & 11 & 19.2 & $14.8-23.0$ & 24.65 \\
$20-30$ & 8 & 6.4 & $3.7-9.1$ & 19.4 \\
$30-40$ & 1 & 2.6 & $0.04-4.9$ & 2.38 \\
$40-50$ & 1 & 3.96 & $3.52-4.4$ & 2.18 \\
Total & 43 & 14.7 & $12.8-16.6$ & 100.0 \\
\hline
\end{tabular}

*Weight in meta-analysis studies is based on the sample sizes and will be accounted from 100 .

Table 3. Geographical distribution of Giardia lamblia in Iran.

\begin{tabular}{lcccc}
\hline Region & Number of studies & Prevalence, $\%$ & $\mathbf{9 5 \%} \mathbf{C l ,} \%$ & Weight $^{*}$ \\
\hline East & 1 & 35.5 & $32.3-38.8$ & 2.29 \\
Center & 14 & 16.9 & $13.0-20.8$ & 32.83 \\
South & 4 & 14.9 & $6.8-23.0$ & 9.26 \\
North & 8 & 13.2 & $6.5-19.9$ & 18.87 \\
West & 16 & 12.2 & $9.3-15.1$ & 36.84 \\
Total & 43 & 14.7 & $12.8-16.6$ & 100.0 \\
\hline
\end{tabular}

promotion, more considering personal health, increased literacy levels and lack of using of the human fertilizers for reinforcement of the agricultural soils. The prevalence of the intestinal parasites was $37.2 \%$ in 2005 in Sivas region, Turkey, in which Giardia vantrobius vermico Laris was the most prevalent infection in the age group of 7-15 years (Celiksoz et al., 2005). In a similar study, the prevalence of Giardia was estimated to be $26.2 \%$ amongst children in Damghan (Heidari, 2003).

In the current study, the rate of Giardia infection was $35.5 \%$ in the eastern regions of the country and $16.9 \%$ in the central areas. In tropical regions and in rural areas in particular, the prevalence of Giardia is expected to be high because of the climatic and health conditions as well as the socioeconomic situation. Despite valuable primary care activities in Iran, the reason behind this finding is due to poor cultural and socio-economic status of people in these regions. People might have not changed the health beliefs and life styles.

In conclusion, increased prevalence of Giardia lamblia in Iran, in spite of preventing strategies and valuable primary health care activities has remained a public health concern amongst children and in the eastern parts of the country nearby Pakistan and Afghanistan borders in particular.

\section{ACKNOWLEDGMENT}

Statistical assistance of Dr. K. Saayemiri and the cooperation of Research Department of Ilam University of Medical Sciences are gratefully appreciated.

\section{REFERENCES}

Abdolali M (2001). Prevalence of intestinal parasitic infections among school children in Yasouj. J. Bring Knowledge 18-17(5):1-9.

Adam R (1991). The biology of Giardia Spp. Microbiol. Rev. 55(4):706732.

Alavinaini S (2000). Prevalence of intestinal parasitic infection among children 5-2 years old and its treatment in the city of Saveh. J. Tehran University Med. Sci. 3(57):48-5.

Amar M (2000). Evaluation of intestinal parasitic infections in elementary schools in Babol city in 1999. Babol. J. Med. Sci. 43:4143.

Aminzadeh Z (2002). Epidemiologic study of intestinal parasitic infections in food vendors in Sanandaj in1999. Ray Med. J. 6(5):1519.

Aminzadeh Z, Nazari M (2004). Prevalence of intestinal parasitic infection among health care applicants in Varamin city in 2003. Tehran J. Med. Sci. 1(3):157-162.

Ashtiani D (2008). Frequency of Giardia and other parasitic infections in pediatric biopsy and aspiration of duodenum and stool samples. J. Pediatr. 103:239-251.

Askari A (2003). Prevalence of intestinal parasite infections among city residents. J. Health School Health Res. Institute. pp. 74-67.

Atashnafas A, Ghorbani R (2006). Prevalence of intestinal parasitic infections and its associated factors in preschool and primary school children in urban areas of Semnan province in 2006. Semnan J. Med. Sci. 8(1):84-75.

Bahadori D (2006). Prevalence of intestinal parasites in Ghaemshahr city in 2004. Mazandaran J. Med. Sci. 15(3):5-15.

Baldo E, Belizario V, De Leon W, Kong H, Chung D (2004). Infection status of intestinal parasites in children living in residential institutions in Metro Manila, the Philippines. Korean J. Parasitol. 142:67-70.

Baruch A, Issac-Renton J, Adam R (1996). Epidemiology of Giardia lamblia. J. Infec. Dis. 174:137-139.

Celiksoz A, Guler N, Guler G, Oztop A (2005). Prevalence of intestinal parasites in three socioeconomically different regions of Sivas Turkey. J. Health Popul. Nutr. 23(2):184-191.

Davami M (2003). Prevalence of intestinal parasitic infections in pregnant women referred to health centers in Arak and associated demographic factors in 2002. Araak J. Med. Sci. pp. 16-11. 
Disney MR-B, Juan D-R, Antonio D-L, Sergio AZ-V, Carmen M, Antonio O, Librado O-O (1998). Secretory immune response to membrane antigens during Giardia lamblia infection in human. Inf. Immun. 66(2):756-759.

Easow J, Mukhopadhyay C, Wilson G, Guha Sand, Jalan B (2005). Emerging opportunistic protozoa and intestinal pathogenic protozoal infestation profile in children of western Nepal. Nepal Med. Coll. J7:134.

Fallahi S (2008). A comparative study of the prevalence of giardiasis in primary school children in Delfan city. Lorestan J. Med. Sci. 9(4):945.

Farahnak A (2002). Prevalence of intestinal parasites in a rural population center in Tabriz. Tabriz J. Med. Sci. 35(49):62-57.

Gharavi M (2006). Seeks to compare the prevalence in school children city Delfan by conventional methods and antigens for Parasitology. Tehran J. Med. Sci. 12(2):4-8.

Gholami S (2005). An intestinal protozoan infections in cattle in rural areas of Mazandaran province in 2004. Mazandaran J. Med. Sci. pp. 60-51.

Ghorbani G (2009). Prevalence of intestinal parasites in the police forces and its association with drinking water. Military Med. 3(10): 166

Guk S, Seo M, Park Y, Oh M, Choe K, Kim J, Choi M, Hong S, Chai J (2005). Parasitic infections in HIV infected patients who visited Seoul National University Hospital during the period 1995-2003. Korean J. Parasitol. 43:1-5.

Hedayati A, Sadraei J, Ghofranipour F (2008). Relationship between the rate of giardiasis and knowledge and practice of prevention in primary school children in south of Tehran. Parasitol. Res. 104:169-171.

Heidari A (2003). Prevalence of Intestinal parasites among children reffered to day-care centers in Damghan-Iran. Iranian J. Publ. Health 32:31-34.

Jahani M, Rezaeian M, Amini M, Daryani N, Ebrahimi M, Montazeri M, Shirzad H (2009). Prevalence of parasites in the small intestine of patients with dyspepsia undergoing upper gastro-intestinal endoscopy in Tehran between 2005-2007. Babol J. Med. Sci. 10(42):60-66.

Khalili M (2005). Prevalence of bacterial agents and Protozoa in 470 stool samples of patients with gastroenteritis referred to Yazd Nikpour hospital. Yazd J. Med. Sci. 1(12):35.

Kheirandish F (2004). Prevalence of intestinal parasites in the city's bakers in Khorramabad in 2003. Lorestan J. Med. Sci. 5(4):43-45.

Mahuti F (2008). Comparison of prevalence rates of childhood Giardiasis in Delfan city. Lorestan J. Med. Sci. 10(4):86.

Mahyar A (1997). Epidemiologic study of Giardia in child care centers in Qazvin in1997. Qazvin J. Med. Sci. 275-263.

Mahyar A (2001). Intestinal parasites in children attending special schools in Qazvin. Qazvin J. Med. Sci. pp. 70-67.

Moghimi M, Asghrshrifl M (2003). Prevalence of parasitic infections in Schoolchildren in Yasoj between 2002-2003. Yasooj J. Med. Sci. $7(26): 80$.

Mohrez M (2005). Prevalence of intestinal parasites among HIV infected individuals in Tehran and Kermanshah. J. Shahid Beheshti University Med. Sci. 8(4): 303.

Mohsenimoghadam F, Shahidizandi B (2008). Prevalence of childhood giardiasis in Rafsanjan in 2005. J. Rafsanjan University Med. Sci. $3(6): 24$.

Molavi G (2008). Prevalence of intestinal parasites in workers. J. Health School Health Res. Institute 5(3):50-43.
Monsef A, Hashemi S, Abbasi M, Taherkhani H, Shalchi Z, Eliasi A (2008). Frequency of intestinal parasites in patients with malignancy, admitted to the Sina Hospital, Hamadan, Iran. Gorgan J. Med. Sci. 9(4):85-90.

Mosallanejad B, Avizeh R, RaziJalali M Alborzi A (2010). Prevalence of Giardia duodenalis Infection in household cats of Ahvaz district, South-West of Iran. J. Iranian Society Parasitol. 5(3):27-34.

Mostafae $G$ (2001). Subterranean water consumption associated with parasitic diseases in the medical diagnostic laboratory referred to Kashan Abozidabad between1999-1998. J. Sci. Res. pp. 60-55.

Nasiri V, Esmailnia K, Karim G, Nasir M, Akhavan O (2009). Intestinal parasitic Infections among Inhabitants of Karaj city between 2006 2008. Korean J. Parasitol. 47(3):265-268.

Rafiee M (2001). Prevalence of parasitic infections in urban schools. Ray Med. J. p. 82.

Razavian T (2003). Intestinal parasitic infections in urban and rural areas next to city center in Mazandaran. J. Health School Health Res. Institute. pp. 49-39.

Rohani S (2001). Prevalence of intestinal parasites in food handlers in Noshahr. Mazandaran J. Med. Sci. pp. 20-15.

Rohani S (2002). Prevalence of intestinal parasites in the rural city of Saari in 2000. J. Zanjan University Med. Sci. pp. 40-33.

Sayyari A, Imanzadeh A (2005). Prevalence of intestinal parasite infections in the Islamic Republic of Iran. East Mediterr Health $\mathrm{J}$. 3:377-383.

Srasyabi K (2001). Prevalence of intestinal parasites in primary school in Bandar Abbas. Hormozgan J. Med. Sci. 7(8):13-16.

Taheri $F$ (2004). SAR's prosperity prevalence of intestinal parasites in children 6 years old in 2002 in Birjand. Isfahan J. Med. Sci. 71(21):35-39.

Taheri F, Namakin K, Zarban A, Sharifzadeh G (2010). Intestinal parasitic infection among school children in South Khorasan province, Iran. J. Res. Health Syst. 11(1).

Taherkhani $H$ (2008). Frequency of intestinal parasites in patients with malignancy. Gorgan J. Med. Sci. 9:85.

Yang G, Wan G, Liu XJ, Shandong P (2003). Summary of the prevalence of Giardia lamblia in Shandong Province. China Trop Med. 